\title{
Estructuras narrativas en relatos cortos y serializados para la web $^{*}$
}

\author{
Diego Montoya Bermúdez \\ Helena García Gómez ${ }^{* * *}$
}

Recibido: 2016-05-21. Enviado a pares: 2016-05-23

Aprobado por pares: 2016-06-06. Aceptado: 2016-06-09

DOI: 10.22395/angr.v15n29a5

\begin{abstract}
Resumen
En el contexto actual la producción y el consumo audiovisual en diversas pantallas ha venido en aumento, gracias al fácil acceso a tecnologías de producción audiovisual y a los bajos costos en la realización, los cuales han posibilitado los intereses narrativos tanto de profesionales como de aficionados, quienes han incursionado en la producción de nuevos formatos, uno de ellos, las series web.
\end{abstract}

No obstante, al momento de la construcción de guiones para dichos formatos, nos encontramos con una notable ausencia de literatura que dé pistas para la creación de estructuras narrativas seriales y sobre todo con temporalidades y apuestas técnicas óptimas para web y dispositivos móviles.

Desde ese orden de ideas el presente texto pretende, a partir de una revisión cuidadosa de algunas series web a la luz de literatura tanto en el ámbito de la narrativa como de la producción audiovisual, proponer claves para la construcción de estructuras narrativas para dicho formato emergente.

Palabras clave: series web, estructuras narrativas, producción audiovisual, guiones audiovisuales.

\footnotetext{
Este artículo presenta resultados de la investigación Estructuras narrativas y claves técnicas para la producción audiovisual en formatos seriales para web y dispositivos móviles, financiada por el Departamento de Humanidades de la Universidad EAFIT y adscrita al Grupo de investigación en Comunicación y Estudios Culturales de la misma Universidad. (COLOMBIA)

** Diego Montoya Bermúdez, comunicador social-periodista de la Universidad Católica Popular de Risaralda, magíster en Comunicación y Creación Cultural, Universidad CAECE en convenido con la Fundación Walter Benjamin, Instituto en Comunicación, Buenos Aires, Argentina.dmonto36@eafit.edu.co.

*** Helena García Gómez, comunicadora social-periodista de la Universidad de Antioquia. Estudiante de la Maestría en Hermenéutica Literaria de la Universidad Eafit. agarci57@eafit.edu.co
} 


\title{
Narrative Structure in Short and Serial Narrations for the web
}

\begin{abstract}
In the current context, audiovisual production and consumption in different screens have been increasing, thanks to the easy access to the audio-visual production technologies and to the low costs in audiovisual productions, which have made possible narrative interests of both professionals and amateurs, who have participated in the production of new formats; one of them, web series.

Nevertheless, at the moment scripts for such formats are being constructed, it has been noticed a notable abscense de literature which provides hints for the creation of serial narrative structure and above all with optimum technical temporalities for the web and mobile devices.

From this statement, this text intends - from a careful revision of some web series at the light of literature in both narrative and audio-visual production, to propose keys for the construction of narrative structures for such an emerging form.
\end{abstract}

Key words: series web, narration structures, audio-visual production, audio-visual scripts 


\section{Introducción}

Desde que apareció la llamada web 2.0 la Internet se convirtió en una plataforma más interactiva pues "la red digital dejó de ser una simple vidriera de contenidos multimedia para convertirse en una plataforma abierta, construida sobre una arquitectura basada en la participación de los usuarios" (Cobo y Pardo, 2007, p.15). Las facilidades de acceso a Internet y la multiplicidad de consumidores e intereses en el contexto de la cultura de convergencia (Jenkins, 2008) han traído consigo nuevas dinámicas de apropiación de este espacio virtual en el que han concurrido medios sonoros, visuales y escritos. Asimismo, el auge de herramientas de fácil acceso para la producción audiovisual y de dispositivos móviles con excelente calidad de reproducción ha permitido la proliferación de productos audiovisuales pensados desde sus inicios para ser visualizados en formatos diferentes a los tradicionales como el cine y la televisión. Prueba de ello es que cada vez se encuentran más en la web repositorios para vídeo como Youtube, Vimeo, Daily Motion, Megavideo, entre otros, que se constituyen como herramientas de fácil acceso en las que se pueden almacenar gratuitamente series y seriales de ficción creadas tanto por profesionales y aficionados, como por empresas y colectivos.

Las series web, entendidas como "todos aquellos seriales de ficción audiovisual creados para ser emitidos por Internet, con una unidad argumental, una continuidad (al menos temática) y más de tres capítulos" (Hernández, 2011, p.92), han crecido de manera considerable tanto en producción como en consumo. Según el informe "TV and Media 2015: The empowered TV and media consumer's influence" realizado por el ConsumerLab de Ericsson', el consumo de series audiovisuales y de entretenimiento en general en Internet ha aumentado en el mundo en más de un $120 \%$ desde 2010. En el caso particular de Colombia, otro informe, el realizado por Comscore sobre el Futuro Digital en 2015, destaca a Latinoamérica como la región del mundo con más aumento en el consumo de contenidos a través de dispositivos conectados a Internet, y Colombia como el país que más visualiza vídeos en youtube con un crecimiento del 118 \% entre el 2014 y el 2015².

Precisamente este crecimiento en el consumo de formatos audiovisuales cortos y serializados está relacionado con un exponencial aumento en la producción, gracias en especial a dos factores: el primero, la proliferación de dispositivos tecnológicos de registro audiovisual de muy buena calidad para la web, y el segundo, los bajos presupuestos que requiere una serie web para su producción, hecho que ha generado que escenarios como el del entretenimiento, la publicidad, el periodismo, la educación, la movilización social, entre otros, estén optando por hacer uso de las series web como formatos alternativos de comunicación.

No obstante, debido a que se trata de un formato audiovisual emergente, la producción ha tomado como base teórica literatura propia del cine y la televisión tradicional, adaptan-

Para ver el informe "TV and Media 2015: The empowered TV and media consumer's influence" Acceder https://goo. gl/U8uS6N.

2 Para ver el informe Futuro Digital en Colombia 2015, acceder http://goo.gl/qgbZJF 
do en la práctica estructuras narrativas autocontenidas ${ }^{3}$ (Long, 2007) de larga duración, a otras serializadas y de cortas temporalidades, ya que no existe suficiente teoría acerca de la producción de series web que facilite los procesos de realización.

La investigación académica sobre webseries y moviseries no está muy extendida a nivel internacional. Existen interesantes publicaciones que se han abocado al impacto de la comunicación móvil o a la comunicación por internet en el contexto de la globalización, en su uso por los movimientos sociales o las transformaciones que estas nuevas tecnologías están generando a nivel de la cognición [Nyíri et al, 2005; Castells et al, 2006; Katz et al, 2008]; sin embargo, existen pocos estudios dedicados a analizar de manera específica lo que está pasando con la industria cultural audiovisual en la actual realidad de multisoportes (Sáez, 2010, p. 2).

Es desde este orden de ideas que este texto, producto de la investigación Estructuras narrativas y claves técnicas para la producción audiovisual en formatos seriales para la web y dispositivos móviles, pretende, a partir de la revisión de una muestra de 12 webseries de distintas partes del mundo, identificar estructuras narrativas recurrentes, apoyados de literatura académica propia del cine y el estructuralismo, con el fin de identificar temporalidades, número de capítulos por temporada y claves del lenguaje audiovisual al momento de producir historias bajo formatos cortos y serializados, y así aportar desde lo académico al emergente escenario de las series web.

Para dar cuenta de ello el texto estará dividido en cuatro partes: una primera, a modo de antecedentes y marco de referencia, en la cual se presentará el contexto en el que se movió la investigación haciendo un breve acercamiento al fenómeno de la producción de series para televisión y dando cuenta de algunos aportes académicos en el campo. En segunda instancia, a manera de método y materiales para la investigación, se presentará el corpus de series web que sirvieron de objeto de estudio y las distintas claves conceptuales que permitieron el análisis de dichos productos. Un tercer momento del texto estará dedicado a los resultados y discusiones evidenciados en el proceso de la investigación, y finalmente, una cuarta parte que servirá para presentar las conclusiones de este trabajo.

\section{Antecedentes y marco de referencia}

Todos seguramente hemos tenido una relación cercana con las series de televisión, pues estos formatos narrativos son tan comunes como el mismo dispositivo de emisión. Desde finales de los años cuarenta, época en la que la televisión se empezó a popularizar en el mundo (Maguregui; Piscitelli; Scolari, 2010), las narrativas audiovisuales buscaron crear formatos que mantuvieran la atención de los espectadores, y gracias al éxito tanto de los folletines policiacos del siglo XIX, como de los formatos radiofónicos de los años 30 del siglo XX, ambos de tipo serializado, generaron una alternativa en el naciente formato audiovisual.

3 Geoffrey Long utiliza el término de productos autocontenidos para referirse a las narrativas que no son secuenciales o serializadas. Una película es autocontenida en tanto tiene un inicio, un nudo y un final y no requiere de varios capítulos donde un personaje continúa el desarrollo de la historia. 
Tanto en la época de la Paleotelevisión como de la Neotelevisión (Eco, 1983), las series audiovisuales han sido formatos de mucho consumo que debido a la secuencialidad narrativa han conquistado múltiples espectadores (Vilches, 1999), gracias a que generan una relación más amplia con los personajes y sus historias. Ahora, en el contexto de la pos-televisión (Piscitelli, 1998) o de la híper-televisión (Scolari, 2010), a la par con la producción de series de televisión tradicional, surgió un formato similar que empezó habitar otras pantallas: las series web, productos audiovisuales emergentes, caracterizados por cortas duraciones pensadas para ser visualizadas en dispositivos móviles en general.

Bajo este epígrafe [el de series web] hemos clasificado lo que vendría a ser el homónimo de las series televisivas en el terreno digital de la red. Es decir, producciones hechas y pensadas para la red que presentan estructura serial, multiplicidad de núcleos narrativos y un despliegue de recursos retóricos específicos que permiten actualizar los avatares de la ficción con el fin de capturar y mantener la atención del receptor capítulo a capítulo (Lloret Romero \& Canet Centellas, 2008, p. 8).

Esta nueva versión de las series audiovisuales ha aumentado de manera considerable su producción en la última década (Sáez Baeza, 2010) (Álvarez, 2011), gracias a que su popularización en tiempo de crisis económica permitió a realizadores jóvenes e independientes crear productos audiovisuales de corta duración en plataformas web gratuitas. Si bien como lo plantea Yolanda Barrasa, estos formatos cortos serializados se conocen desde finales del siglo XX, fue con la aparición de diferentes plataformas web como garantizaron su permanencia y consumo.

Su popularidad, en principio, se debe a la brevedad, y a que se pueden ver en cualquier lugar y en cualquier dispositivo. Sin embargo, millones de nativos digitales que no pueden concentrarse más de diez minutos en una sola cosa, les han exigido contundencia gracias a que cuentan con dispositivos móviles y una buena conexión de datos, y es allí donde las series web han respondido a las necesidades de ese nuevo espectador (Barrasa, 2015, p. 16).

Justamente esta respuesta efectiva de las series web a los nuevos consumidores fue la que permitió el crecimiento sustancial en producciones, que ahora, gracias a que cuentan con un público cautivo, han empezado a ser producidas también por empresas de comunicación y publicidad con mucha experiencia, quienes han visto en estas un formato alternativo y muy efectivo para escenarios como el publicitario, debido a que son "un dispositivo maravilloso para establecer, generar y fidelizar audiencias" (Barrasa, 2015, p. 17).

Muchas empresas relacionadas con el mundo de la comunicación han visto una gran oportunidad en Internet como soporte para distribuir contenidos implicando a importantes y destacadas marcas comerciales incluyendo nuevas formas de publicidad o patrocinio (Peñafiel y Echegaray, 2012, p.10). 


\section{Metodología y materiales para la investigación}

\subsection{Nociones conceptuales}

Para llevar a cabo la investigación sobre estructuras narrativas y claves técnicas para la producción de formatos cortos serializados para la web, se recurrió, en primera instancia, a un material bibliográfico propio de la teoría cinematográfica y el estructuralismo con los cuales abordar el objeto de estudio: las series web.

Fue así como a partir de Syd Field se asumió la revisión del guion audiovisual, teniendo en cuenta que el autor norteamericano tiene como objeto de estudio el cine y en especial el guion para largometrajes; no obstante, este acercamiento a la narrativa audiovisual permitió ver en primera instancia el guion de las series a partir de los planteamientos de Doc Comparato, quien lo plantea como una doble estructura: por un lado, "una macro o estructura general que reúne todo el serial con sus distintos capítulos, ly por el otrol una micro estructura que se refiere a cada uno de los capítulos de manera independiente" (1999, p. 32).

Por otra parte, en lo que refiere al análisis estructural del relato, este se abordó a partir de diferentes autores, uno de ellos, Claudé Bremond, quien plantea que un relato debe iniciar con una secuencia elemental, en la cual se establecen ciclos de mejoramiento y degradación del personaje principal en pos del desarrollo de la historia:

Es una primera agrupación de tres funciones o tres fases obligadas de todo proceso de relato: a) una función que abre la posibilidad del proceso en forma de conducta a observar o de acontecimiento a prever; b) una función que realiza esta virtualidad en forma de conducta o de acontecimiento en acto y c) una función que cierra el proceso en forma de resultado alcanzado (Bremond, 1974, p. 87).

En línea con los aportes estructuralistas, otros de los abordajes hechos dentro de la investigación fueron los referidos a las nociones de Joseph Campbell, Christopher Vogler y Lauro Zavala con relación a las acciones del personaje principal, en una suerte de modelo de estructura mítica denominada Viaje del héroe (Campbell, 1972) y que para efectos de la producción de series web resultó fértil, en tanto permitió la secuencialidad y el desarrollo del personaje a lo largo de toda la macro estructura.

El modelo del viaje del héroe tiene un alcance universal, ocurre en todas las culturas y en todas las épocas... Los personajes recurrentes en el mundo de los mitos tales como el joven héroe, el anciano o anciana sabios, la figura que cambia de aspecto, el antagonista sombrío y escurridizo, son idénticos a esas figuras con presencia constante en nuestros sueños y fantasías. Es por eso por lo que los mitos y muchas de las historias elaboradas según el modelo mitológico resultan convincentes y nos presentan una ilusión de la verdad psicológica (Vogler, 2002, p. 32-33).

Estos enfoques, cinematográfico y estructuralista, sirvieron de marco para analizar los diferentes productos serializados hechos para la web que fueron objeto de estudio y que a continuación serán descritos. 


\subsection{Objeto de estudio: breve descripción}

Como se ha venido anunciando a lo largo del texto, uno de los intereses fundamentales para la investigación fue el de analizar producciones audiovisuales de tipo serial para la web, con el fin de identificar cómo se dan las estructuras narrativas, teniendo en cuenta que una de las características que distinguen a las series web de la televisión tradicional es que no existe un consenso en el número de capítulos que pueda tener una serie web por temporada. Si bien en televisión tradicional también se pueden encontrar series con variaciones en el número de capítulos, hay algunos estándares establecidos como series de 13 capítulos.

Ahora bien, para la investigación se eligieron 12 series web distintas tanto en temáticas como en número de capítulos por temporada; esto con el fin de identificar cómo se desarrollan las estructuras narrativas dependiendo el número de capítulos que componen una temporada. Por otra parte, para la elección de las distintas series objeto de estudio, se asumieron cuatro criterios: uno, que las series web escogidas tuviesen una estructura dramática de tipo serial. Según Doc Comparato hay que hacer distinción entre lo que es una serie y un serial; por un lado, las series plantean "un mismo personaje que vive historias diferentes, mientras que el serial muestra a personajes viviendo una historia en 2 , 4, 6 o hasta 20 capítulos" (1999, p. 32). Es de anotar que para el caso de las series web se mantuvo la nominación de series, aunque para efectos de la investigación se analizaron productos con estructuras dramáticas de tipo serial. El segundo criterio de selección que tuvimos en cuenta fue el de series hechas para la web, esto implica que sus capítulos tuvieran una corta duración con un máximo de 15 minutos por capítulo. En tercera instancia se consideró el hecho de que las series web a analizar tuvieran por lo menos una temporada finalizada. Esta clasificación se hizo con la pretensión de poder rastrear elementos repetitivos en la construcción de una historia completa contada en un número determinado de capítulos. Cada temporada fue vista por consiguiente, como un producto, en la medida que funciona como el todo de una historia (macroestructura). Un criterio final que se tuvo en cuenta para la selección del objeto de estudio fue que las series web contaran con un impacto significativo avalado por un amplio número de seguidores o simpatizantes, o por su nivel de calidad reconocido a través de premios o galardones.

Cabe anotar que la diversidad de las series escogidas también radicó en los equipos de producción, los cuales son muy heterogéneos: uno, profesionales de la industria audiovisual que quieren darle continuidad a algún producto de la televisión tradicional en una forma de transmedialidad (Scolari, 2010); dos, empresas de comunicación especializada que producen series por demanda de una compañía como estrategia publicitaria; tres, estudiantes y docentes de carreras afines a la comunicación, el cine y la industria audiovisual como forma de dar a conocer su trabajo, y cuatro, productos realizados por colectivos de aficionados a la producción audiovisual.

A continuación, la tabla 1 presenta una ficha técnica con las series web analizadas durante la investigación; esta selección fue, a la vez, clasificada en grupos por número de capítulos, así: grupo 1, series con 6 o menos capítulos; grupo 2, series de 7 a 11 capítulos; 
grupo 3, series con 12 o más capítulos. Una de las series que tuvo varias temporadas fue The Guild; a esta se le analizaron dos temporadas diferentes porque el número de capítulos varió y eso permitía revisar cómo se comportó la estructura narrativa en un mismo producto con diferencia en el número de capítulos.

Tabla 1: Ficha técnica de webseries analizadas

\begin{tabular}{|c|c|c|c|c|c|}
\hline Grupo & Serie & Capítulos & Temporada & Director o grupo realizador & País \\
\hline \multirow{6}{*}{1} & The Oath & 3 & 1 & AMC Studios & USA \\
\hline & Cold Storage & 4 & 1 & AMC Studios & USA \\
\hline & Diario del apocalipsis & 6 & 1 & Yolanda Barrasa & España \\
\hline & Del otro lado & 6 & 1 & Diego Durán & Colombia \\
\hline & Beauty Inside & 6 & 1 & Toshiba - Intel & USA \\
\hline & Torn Apart & 6 & 1 & AMC Studios & USA \\
\hline \multirow{4}{*}{2} & Ruta 66 & 7 & 1 & Sergi Cervera & España \\
\hline & Compulsion & 8 & 1 & Nathan Atkinson & USA \\
\hline & Desubicados & 8 & 1 & $\begin{array}{l}\text { Fernando Pérezgil \& Yose- } \\
\text { line Hoffman }\end{array}$ & México \\
\hline & The Guild & 10 & 1 & Jane Selle Morgan & USA \\
\hline \multirow{3}{*}{3} & Susana y Elvira & 12 & 1 & Mimosa TV & Colombia \\
\hline & Déjà Vu & 12 & 1 & $\begin{array}{l}\text { Juan Francisco Pérez } \varepsilon \\
\text { Edwin Herrera }\end{array}$ & Colombia \\
\hline & The Guild & 12 & 2 & Sean Becker & USA \\
\hline
\end{tabular}

\section{Resultados y discusiones}

Con el fin de lograr un análisis más claro de las diferentes series web seleccionadas y para cotejarlas a la luz de los aportes teóricos descritos en el punto anterior y que se desarrollarán en este, se realizaron unas matrices que permitieron revisar tanto las estructuras narrativas como los aspectos del relato concernientes a los distintos capítulos.

Así, una primera matriz dirigida a la macroestructura narrativa tomó como base la fragmentación terciaria de la estructura dramática propuesta por Aristóteles en su Poética, la cual fundamenta la narración en tres actos: inicio, nudo y desenlace. Por efectos prácticos y más pertinentes con esta investigación por su relación con el campo audiovisual, se abordó el paradigma presentado y definido por Syd Field como "un modelo, un ejemplo, un esquema conceptual del aspecto que tiene un guion" (2005, p. 21), el cual permitía comprender mejor lo que sucedía en el transcurso de cada uno de los tres actos, a partir de un planteamiento, una confrontación y una resolución. 
Field establece cada acto como "una unidad o bloque de acción dramática" (2005, 22). Es por ello que en el planteamiento o Acto I se instala la historia a través de la presentación de los personajes principales, la premisa dramática, la creación de la situación y la disposición de escenas y secuencias que elaboran y desarrollan la información sobre la historia. En el II Acto, conocido como confrontación, el protagonista se enfrenta a obstáculos y conflictos que deben ser resueltos o superados en aras de satisfacer su necesidad dramática. Syd Field deja muy claro cómo esta unidad dramática constituye el culmen de la historia pues "el drama es conflicto; sin conflicto no hay acción; sin acción no hay personaje; sin personaje no hay historia, y sin historia no hay guion" (2005, p. 25). Finalmente el Acto III da cuenta de cómo se resuelve la historia. El contexto dramático de esta unidad narrativa es la resolución, entendida como "la solución" a la necesidad dramática del personaje. Asimismo, dentro del paradigma presentado por Field existen dos puntos de giro o plot points, los cuales están presentes al final de los dos primeros actos. Para el autor norteamericano un plot point "es un incidente, episodio o acontecimiento que se "engancha" a la acción y la hace tomar otra dirección [...]; puede ser cualquier cosa: un plano, unas palabras, una escena, una secuencia, una acción, lo que sea que haga avanzar la historia" (p. 26).

Ya en lo que respecta a la revisión de las series web objeto de estudio, en Susana y Elvira (grupo 3, tabla 1), por ejemplo, se identificaron como protagonistas a dos amigas cuya necesidad dramática en ambas fue la misma: encontrar el amor, y para generar conflicto los personajes asumieron estados emocionales diferentes respecto a las relaciones sentimentales. Así, Susana al no olvidar a su ex, permanentemente sostuvo relaciones sexuales ocasionales sin involucrarse sentimentalmente, mientras que Elvira acababa de terminar una larga relación con su novio. En la primera temporada de esta serie, se identificó el plot point del primer acto en el capítulo 5 cuando se introdujo un personaje llamado Camilo. Dicho personaje se constituyó como un "punto de giro" en la historia personal de Susana, pues se convirtió en su objeto de amor e inició con él una relación monógama. El segundo plot point, ubicado en el acto II, se correspondió con la historia de Elvira, pues en el capítulo 10, después de una serie de frustraciones del personaje, se vio cómo ella desempacó una caja que le trajo recuerdos de su relación y decidió "salir" de las cosas que le recordaban a su exnovio.

Volviendo a la revisión bibliográfica, a la par con los planteamientos de Syd Field en torno al desarrollo de una historia a través de los actos narrativos, es importante tener en cuenta los aportes de Barthes (1974) con relación a las funciones que debe tener un relato para que este progrese. En términos de la teoría cinematográfica, Marimón (1960) propone estas funciones como plants y payoff; las primeras, dedicadas a la instalación de acciones que luego, en las segundas, deben ser resueltas. De esta manera todas las historias en sus distintos actos deben generar indicios (plants) que luego son resueltos (payoff), eso con el fin de que el relato progrese y se dé cuenta del cumplimiento del conflicto con total naturalidad.

Estos elementos fueron evidenciados en todas las series web analizadas sin importar el número de capítulos; asimismo, se identificó que entre menos capítulos tuviera la 
serie web, más plants se debían instalar, con el fin de generar una rápida resolución. En esa misma vía, los productos serializados con menos capítulos tuvieron la obligación de pasar al acto II en un máximo de dos capítulos con el fin de mantener la atención del espectador. Para el caso de una serie de más capítulos, como sucede en Susana y Elvira, el espacio de instalación del mundo ordinario y las primeras etapas del viaje del héroe pueden demorarse un poco más.

Una de las series web analizadas del grupo 1 que no desarrolló con celeridad el primer acto y posteriormente evidenció problemas para el cerramiento del relato fue Diarios del apocalipsis, la cual dejó en el nivel de las funciones una sucesión de plants que no fueron resueltos. En el caso particular de esta serie, el relato se concentró en desarrollar en el primer acto el mundo ordinario y el llamado a la aventura del personaje (ver tabla 2), pero no evolucionó en las demás etapas para cruzar el umbral que lo llevara a la resolución. Si bien la serie cumplió con un arranque In medias res ${ }^{4}(\mathrm{Eco}, 1997)$ como es recomendado para series de pocos capítulos, al dejar plants sin resolver no generó la sensación de un relato completo.

Por otra parte, y retomando el ejemplo de la serie Susana y Elvira, se identificó que en la primera temporada existieron varios capítulos que se conectaron entre sí a partir del uso de diversos plants, los cuales fueron resueltos en capítulos posteriores. Esta estrategia ayudó a mantener la conexión de los espectadores a lo largo de toda la macroestructura y es conocida en el contexto de los relatos serializados como cliffhanger, la cual, según Codes (2013), funciona como la manera en que el autor mantiene la expectativa del espectador. Prueba de ello se observa en los capítulos 4 y 5 (ubicados dentro del acto I), 7 y 8 (en el acto II) y 11 y 12 (en el acto III). Dichos episodios retoman el plant dejado en el capítulo inmediatamente anterior, es decir, el indicio sirve como conector de la historia al ser resuelto - payoff-en el siguiente. La estrategia de usar los plants y payoff como cliffhanger fue común en la mayoría de las series analizadas y que dejaron la sensación de generar relatos completos.

En lo que refiere a la estructura general y el cumplimiento de los actos, en las series más cortas analizadas (Grupo 1, tabla 1) como Cold storage y The Oath, se identificó que cada capítulo se correspondía con un acto, mientras que en las series con mayor número de capítulos (12), la historia se concentró en el planteamiento y la confrontación, es decir, se presentaban los personajes, su intención dramática, su conflicto y todo se resolvía finalmente en el tercer acto que se constituía como el clímax en la narración.

Esta división básica de la estructura dramática presentada por las series da cuenta de la macroestructura, en otras palabras, de cómo se configura el relato en términos generales. Es clave anotar que sin importar el número de capítulos de una serie web, siempre

$4 \quad$ En términos de Umberto Eco "[es] el principio de la narración in medias res, el inicio mediante un diálogo, la rápida insistencia sobre una historia individual en vez de general, y sobre todo, inmediatas marcas de ironía". (Eco, 1997, 138) Esta estrategia narrativa es clave para la producción de webseries, porque ahorra tiempo en presentaciones y desarrollo de los mundos posibles instalados en el relato, y pone las cartas de juego de manera ágil y en pro de una efectiva resolución. 
se deben cumplir los tres actos, sea porque cada acto corresponde a un capítulo en una serie web de solo tres, o porque cada acto se desarrolle en varios capítulos.

Siguiendo esta misma línea, respecto a elementos más puntuales que permiten entender la historia, se construyó una matriz para el análisis de cada capítulo de la serie con base en postulados teóricos de Claude Bremond y Lauro Zavala. Bremond, haciendo un análisis estructural del relato (1974), da cuenta de una lógica de posibles narrativos los cuales parten del proceso de mejoramiento o degradación que pueda sufrir el personajehéroe de la historia. Un proceso similar de mejoramiento o degradación propone Lauro Zavala (2007) trayendo a colación los planteamientos de Campbell en la idea del "viaje del héroe", la cual para el autor norteamericano consiste en el "seguimiento a una multitud de figuras heroicas a través de las etapas clásicas de la aventura universal" (1972, p. 28).

Precisamente este viaje que realiza el héroe sirvió como complemento en el análisis de la macroestructura de las series web, en tanto, a partir de los planteamientos antes mencionados se construyó una segunda matriz (tabla 2) que permitió ver con claridad los actos narrativos en una temporada completa.

Tabla 2. Elementos estructurales de la historia: Matriz elaborada a partir de la lectura al modelo propuesto por Lauro Zavala Alvarado sobre los elementos de análisis cinematográfico

\begin{tabular}{|c|c|c|c|}
\hline \multicolumn{4}{|c|}{ NARRACIÓN (Elementos estructurales de la historia)* } \\
\hline \multicolumn{4}{|c|}{ ¿Qué elementos permiten entender la historia? } \\
\hline \multicolumn{3}{|c|}{ Elementos de la estructura mítica } & Capítulo \\
\hline \multirow{5}{*}{$\overrightarrow{0}$} & Mundo ordinario: & Presentación del héroe y su falta & 1 \\
\hline & Llamado a la aventura: & Tentanción y reconocimiento & 2 \\
\hline & Rechazo de la llamada: & Mostrar lo formidable del reto & 3 \\
\hline & Encuentro con mentor: & Protección, prueba o entrenamiento & 4 \\
\hline & Cruzamiento del umbral: & momento de decisión, acto de fe. & 5 \\
\hline \multirow{6}{*}{ 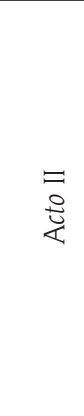 } & Pruebas, aliados, enemigos: & Compañía, sombra, rival & 6 \\
\hline & $\begin{array}{l}\text { Acercamiento a la cueva más } \\
\text { remota: }\end{array}$ & hilo de complicaciones & 7 \\
\hline & \multicolumn{3}{|c|}{ INICIO DE LA CRISIS CENTRAL (Punto de no retorno) } \\
\hline & Reto supremo: & $\begin{array}{l}\text { Los héroes deben morir para poder } \\
\text { renacer }\end{array}$ & 8 \\
\hline & Recompensa: & epifanía, celebración, iniciación & 9 \\
\hline & Jornada de regreso: & Contraataque o persecusión & 10 \\
\hline \multirow{3}{*}{ 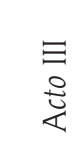 } & Resurección: & Duelo a muerte y dominio del problema & 11 \\
\hline & \multicolumn{3}{|l|}{ CLÍMAX } \\
\hline & Regreso con elíxir: & Prueba, sacrificio, cambio & 12 \\
\hline
\end{tabular}


Como se pudo observar en la tabla 2, una serie de doce capítulos puede responder de manera directa con el modelo del viaje del héroe; sin embargo, cuando se trata de series de menos capítulos hay que prescindir o mezclar etapas. De esta manera, revisando los productos objeto de estudio correspondientes a los grupos 1 y 2 de la tabla 1 , se encontró que hubo una serie de etapas que siempre se cumplen y otras que van siendo descartados a medida que la serie tiene menos capítulos. Las etapas que siempre aparecen sin importar el número de capítulos se definieron como constantes, y las que son prescindibles o mezclables, como variables.

Para el caso de las constantes todas las series web analizadas demostraron este tipo de funciones en la historia: presentación del héroe y su falta (capítulo 1); en términos de Bremond, se presenta el personaje y el fin alcanzable. El final también permanece constante, pues debido a que es la intención dramática del personaje la que marca el conflicto en la historia, la transformación del mismo se da al concretar o no dicha intención; la retribución: recompensa y venganza, y el regreso con elíxir se constituyen como los elementos estructurales que dan cuenta de ese proceso de transformación final del personaje-héroe. Los otros elementos, los denominados variables, se pueden presentar de manera deliberada en el relato mismo, aunque se encontraron ciertas similitudes en las series como que la eliminación del adversario, las pruebas, aliados y enemigos se suelen presentar en el acto 2 como una forma de reavivar la trama o darle un viraje, un cambio de suerte al personaje principal.

En las series cortas de 6 o menos capítulos (grupo 1, tabla 1) se imponen ciertas funciones en el desarrollo de la historia como el cumplimiento de la tarea, la obligación, la negociación, la agresión y la intervención del aliado. Por otro lado, en las series de 12 capítulos suelen presentarse todas las funciones detalladas en la matriz, es decir, la historia gira alrededor de esos ejes de acción que se constituyen como rasgos tradicionales dentro de las estructuras narrativas.

Para identificar cada una de las series analizadas y cómo se corresponden con la propuesta del viaje del héroe se diseñó la tabla 3, que da cuenta con claridad de las etapas constantes y variables, dependiendo el número de capítulos de cada macroestructura.

\section{Conclusiones}

Las series web en la actualidad son un fenómeno audiovisual emergente cuya dinámica opera bajo ciertos criterios que varían respecto a los formatos audiovisuales tradicionales. Solo con mencionar algunas de estas características se puede evidenciar que nos encontramos ante un fenómeno cuyas reglas de juego están aún en construcción. Entre ellas, se destaca que las series web han respondido a nuevas formas de consumo, propias de un tipo de usuario diferente que consume a la carta, en diversas pantallas y con intenciones de mayor interacción; estas actitudes activas del usuario favorecen otras interacciones e hibridaciones en diferentes ámbitos como el de la producción y el mercadeo. Es así como no es ajeno asociar el término crowdfunding (recolección de recursos por Internet), a 


\begin{tabular}{|c|c|c|c|c|c|c|c|c|c|c|c|}
\hline \multirow{10}{*}{ 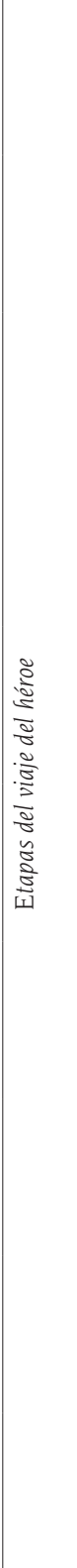 } & & & & & & & & & & 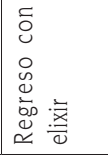 & 으 \\
\hline & & & & & & & & & & 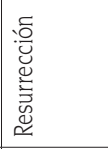 & $a$ \\
\hline & & & & & & & & 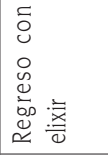 & 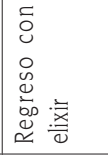 & 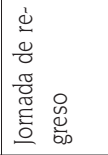 & $\infty$ \\
\hline & & & & & & & 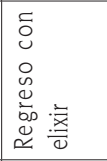 & 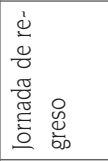 & 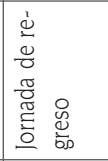 & 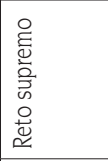 & $r$ \\
\hline & & & 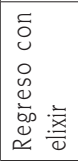 & 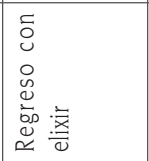 & 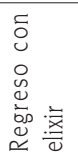 & 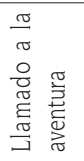 & 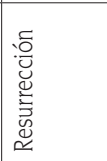 & 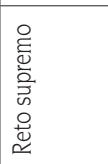 & 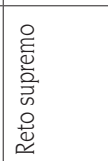 & 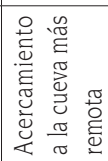 & 0 \\
\hline & & & 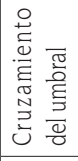 & 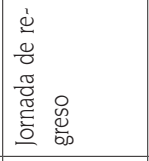 & 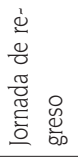 & 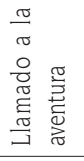 & 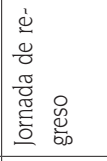 & 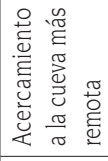 & 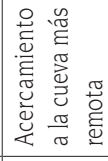 & 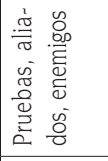 & in \\
\hline & & 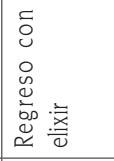 & 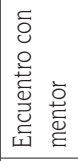 & 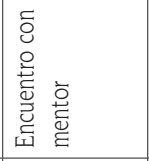 & 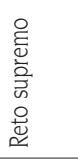 & 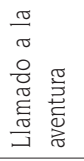 & 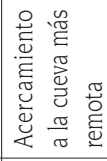 & 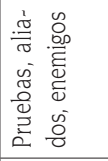 & 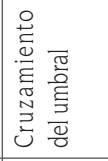 & 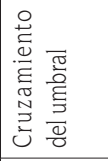 & $\diamond$ \\
\hline & 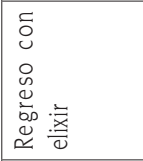 & 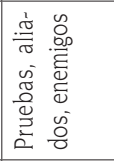 & 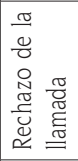 & 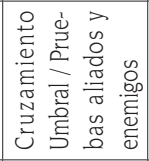 & 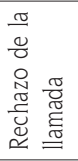 & 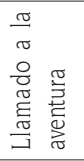 & 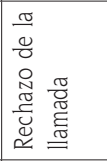 & 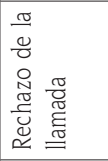 & 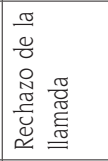 & 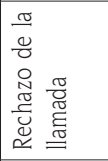 & $m$ \\
\hline & 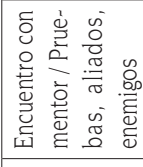 & 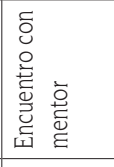 & 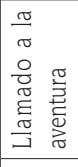 & 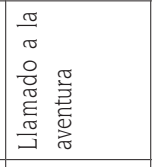 & 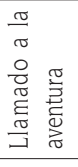 & 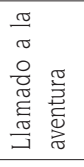 & 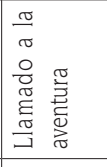 & 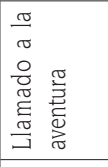 & 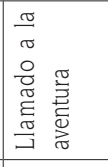 & 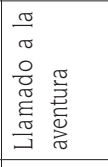 & $\sim$ \\
\hline & 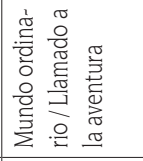 & 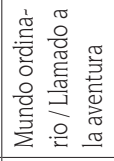 & 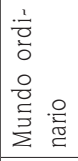 & 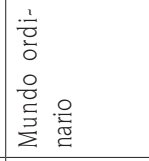 & 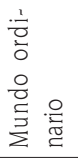 & 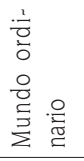 & 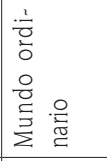 & 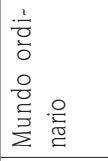 & 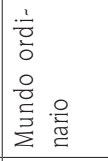 & 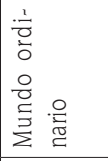 & - \\
\hline है & $n$ & $\nabla$ & 0 & 0 & 0 & 0 & $r$ & $\infty$ & $\infty$ & 으 & \multirow{3}{*}{ 鹤 } \\
\hline \multirow[t]{2}{*}{ 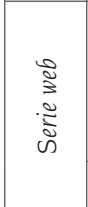 } & 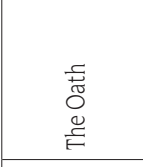 & $\begin{array}{l}0 \\
00 \\
\frac{0}{0} \\
0 \\
0 \\
0 \\
0\end{array}$ & 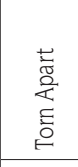 & 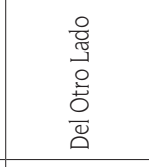 & 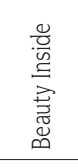 & 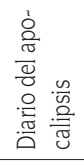 & 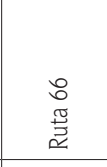 & 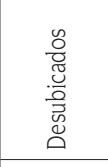 & $\begin{array}{l}\frac{.0}{0} \\
\frac{0}{\bar{n}} \\
\overline{\bar{g}} \\
0\end{array}$ & 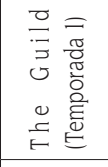 & \\
\hline & - & $\sim$ & $n$ & $\nabla$ & in & 0 & r & $\infty$ & $a$ & 으 & \\
\hline
\end{tabular}


la realización de productos audiovisuales por Internet y a otras iniciativas sociales, como fue el caso de la primera temporada de The Guild, donde la participación activa de los usuarios-fans fue la que permitió la supervivencia del proyecto.

Justamente, por el hecho de ser un formato emergente y que sus reglas de producción se siguen definiendo, es importante hacer énfasis en las diferencias con relación a los seriales de la televisión tradicional; de hecho, plataformas tecnológicas como Netflix, a pesar de hacer uso de la web para la distribución de contenidos, no emite series web, porque sus producciones no son cortas en términos de temporalidades, y los tiempos de cada capítulo de una misma serie son constantes. En el caso que compete a esta investigación se identificó que una misma serie web puede contener capítulos con duraciones diversas las cuales fluctúan entre tres y quince minutos, y estas variaciones, para efectos de la producción audiovisual para la web, ayudan al momento de la construcción de guiones, en tanto en una serie web de pocos capítulos, a mayor tiempo de un webisodio, más posibilidad de imbricar etapas variables del viaje del héroe; y la flexibilidad de las series web con el número de capítulos justifica la estrategia de definir etapas constantes y variables.

Precisamente con relación a las etapas constantes, a lo largo de la investigación se comprobó que para dar cuenta de ellas se requiere, como lo anotamos con anterioridad, de por lo menos tres capítulos, en tanto estas etapas constantes se relacionan directamente con los actos. Precisamente en la revisión del corpus para el análisis, nunca se encontró ninguna serie web menor a tres capítulos.

Para terminar, la investigación ayudó a plantear claves narrativas y estructurales posibles de utilizarse para la producción de series web. El alto crecimiento del formato, tanto en su producción como en su consumo, nos lleva desde la academia a revisar las formas de construcción de este tipo de relatos cortos y serializados, los cuales no son comunes para la enseñanza tradicional en áreas audiovisuales. Este proceso de pesquisa planteó algunos puntos de arranque posibles de ser usados para la construcción de los relatos, pero abre otras líneas que también tienen que ver con este objeto de estudio, algunas de ellas, las que refieren a las diferencias del lenguaje audiovisual y los aspectos técnicos propios de una producción para pantallas de pequeñas dimensiones, pero esto es un tema que continuaremos trabajando.

\section{Referencias bibliográficas}

Álvarez Monzonillo, J. (2011) La televisión etiquetada: nuevas audiencias, nuevos negocios. Barcelona: Editorial Ariel.

Barrasa, Yolanda (2015) Series web: revolución creativa en las redes de largo alcance. En Revista Arcadia. N. ${ }^{\circ}$ 117. Bogotá: Publicaciones Semana.

Barthes, R. (1974). Introducción al análisis estructural de los relatos. En E. Verón (Dir.) Análisis estructural del relato. Argentina: Tiempo Contemporáneo.

Bremond, C. (1974). La lógica de los posibles narrativos. En E. Verón (Dir.) Análisis estructural del relato. Argentina: Tiempo Contemporáneo. 
Campbell, J. (1972). El héroe de las mil caras. Psicoanálisis del mito. México: Fondo de Cultura Económica.

Cobo, C. E Pardo, H. (2007) Planeta Web 2.0.Inteligencia colectiva o medios fast food. Grup de Recerca d'Interaccions Digitals, Universitat de Vic. México DF: Flacso.

Codes, M. (2013) Intriga y Suspense, el gancho invisible. Madrid: ALBA

Comparato, D. (1999) La serie dramática. En L. Vilches (Comp.) Taller de escritura para televisión. Barcelona: Gedisa.

Comscore (2015) Futuro Digital Colombia 2015 [Informe]. Recuperado el 5 de mayo de 2016 Disponible en http://goo.gl//k5BGA

Consumer Lab, Ericsson (2015) TV and Media 2015: The empowered TV and media consumer's influence [Informe]. Recuperado el 5 de mayo 2016. Disponible en: https://goo.gl/U8uS6N

Coronado Ruiz, C (2011, diciembre). Series web: ¿el futuro de la televisión? Ponencia presentada en el Tercer Congreso Internacional Latina de Comunicación Social, Tenerife, España.

Eco, U. (1983) La Estrategia de la Ilusión. Barcelona: Lumen.

Eco, U. (1997) Seis paseos por los bosques narrativos. Barcelona: Lumen.

Field, S. (2005) Screenplay. The foundations of screenwriting. New York: Delta.

García Avilés, J. (2009) Conferencia de clausura. La otra televisión posible. EN: León, Bienvenido. Transformar la Televisión: otra televisión posible. Sevilla: Comunicación Social, ediciones y publicaciones.

Hernández, P. (2011). Las webseries: Evolución y características de la ficción española producida para Internet. Revista Faro, 7(13), 91-100.

Jenkins, H. (2008). Convergence Culture: Where Old and New Media Collide. New York: New York University Press.

Long, G. (2007) Transmedia Storytelling. Business, Aesthetics and Production at the Jim Henson Company. Massachusetts: MIT.

Marimón, J. (1960) El montaje cinematográfico: del guión a la pantalla. Barcelona: Comunicación Activa Audiovisual \& Universidad de Barcelona.

Lloret Romero, N. E Canet Centellas, F. (2008) Nuevos escenarios, nuevas formas de expresión narrativa: La Web 2.0 y el lenguaje audiovisual. Recuperado en Abril de 2016 len línea]. "Hipertext.net", núm. 6.

Maguregui, C; Piscitelli, A y Scolari, C. (2010) Lostología. Estrategias para entrar y salir de la isla. Buenos Aires: Editorial Cinema

Peñafiel, C. y Echegaray, L (2012, Enero). El fenómeno de los "microcontenidos": riesgo o acierto en una sociedad "hipertecnologizada". Ponencia presentada en el Tercer Congreso Internacional Asociación Española de Investigación de la Comunicación, Tarragona, España.

Piscitelli, A. (1998) Post/Televisión. Ecología de los medios en la era de Internet. Buenos Aires: Paidós Contextos.

Sáez Baeza, C. (2010, febrero). Series para móviles y series por Internet: el lenguaje de las nuevas pantallas y su aterrizaje en la televisión española. Ponencia presentada en el Segundo Congreso Internacional Comunicación y Desarrollo en la Era Digital, Málaga, España.

Scolari, C. (2010) Narrativa transmediática, estrategias cross-media e hipertelevisión. En C. Maguregui; A. Piscitelli y C. Scolari (Comp.) Lostología. Estrategias para entrar y salir de la isla. Buenos Aires: Editorial Cinema 
Vilches, L.(1999) Taller de escritura para televisión. Barcelona: Gedisa.

Vinader, R., Abuín, N. y García, A (2011). El impacto de la Web 2.0 en los perfiles profesionales audiovisuales. Revista Telos, 87, 73-83.

Vogler, C. (2002). El viaje del escritor. Las estructuras míticas para escritores, guionistas, dramaturgos y novelistas. Buenos Aires: MaNonTroppo .

Zavala Alvarado, L. (2007). Manual de análisis narrativo. Literario, cinematografía, intertextual. México: Ed. Trillas. 